\title{
Características de carcaça de cordeiras Pantaneiras abatidas com diferentes espessuras de gordura subcutânea
}

\author{
[Carcass characteristics of Pantaneiro lambs slaughtered with different \\ subcutaneous fat thickness] \\ N.H.A.P. Mora ${ }^{1}$, F.A.F. Macedo ${ }^{2}$, A.A. Mexia ${ }^{3}$, F.B. Dias-Senegalhe ${ }^{2}$, \\ E.Q. Oliveira ${ }^{2}$, A.C. Radis ${ }^{2}$ \\ ${ }^{1}$ Aluno de pós-graduação - Universidade Estadual de Maringá - UEM/PPZ - Maringá, PR \\ ${ }^{2}$ Universidade Estadual de Maringá - UEM/PPZ - Maringá, PR \\ ${ }^{3}$ Universidade do Estado do Mato Grosso - UNEMAT - Tangará da Serra, MT
}

\begin{abstract}
RESUMO
Foram utilizadas 24 cordeiras do grupo genético Pantaneiro, abatidas com 2,0; 3,0 e 4,0 mm de espessura de gordura, avaliadas por ultrassonografia no Longissimus dorsi. Avaliaram-se características quantitativas de carcaça, do lombo e índice de musculosidade da perna. As cordeiras receberam ração completa misturada calculada para ganho de peso diário de $0,300 \mathrm{~kg}$. Foram realizadas avaliações por ultrassonografia (entre a $12^{\mathrm{a}}$ e a $13^{\mathrm{a}}$ costela) e pesagens a cada 14 dias, sendo que os abates ocorriam à medida que as cordeiras atingiam a espessura de gordura pré-determinada: 2,0; 3,0 e 4,0 mm. Não houve efeito para rendimento verdadeiro da carcaça, rendimento comercial da carcaça e índice de compacidade da perna. Foi observado maior rendimento do lombo para cordeiras abatidas com 4,0 mm de espessura de gordura subcutânea. As espessuras de gordura subcutânea não influenciaram a área de olho de lombo, porém animais com 4,0 mm apresentaram maiores medidas. Foram observadas diferenças nas proporções de músculo, osso e gordura da perna entre as espessuras de gordura subcutânea avaliadas. A musculosidade da perna não foi influenciada com o aumento da espessura da gordura subcutânea. Com base neste estudo e também com as exigências do mercado consumidor, recomenda-se o abate de animais com aproximadamente 3,00 $\mathrm{mm}$ de gordura.
\end{abstract}

Palavras-chave: fêmeas, musculosidade, rendimento, ultrassom

\begin{abstract}
Twenty-four female lambs, all from the Pantaneiro genetic group, slaughtered with 2.0; 3.0 and $4.0 \mathrm{~mm}$ of subcutaneous fat thickness measured by ultrasound in Longissimus dorsi, were used. We evaluated quantitative carcass traits, loin and leg muscularity. The lambs were fed with total ration calculated for daily weight gain of $0.3 \mathrm{~kg}$. Ultrasound evaluations (between the $12^{\text {th }}$ and the $13^{\text {th }}$ ribs) and weightings were carried out each 14 days; the slaughter occurred as the lambs reached the predetermined fat thickness: 2.0, 3.0 and $4.0 \mathrm{~mm}$ regardless of body weight. There was no effect for real income carcass, commercial carcass yield and index of leg compactness. Higher yield of loin for lambs slaughtered at 4.0 mm fat thickness was observed. The thickness of subcutaneous fat did not affect loin eye area, but animals with $4.0 \mathrm{~mm}$ had higher measures. There were differences in the proportions of muscle, bone and fat leg between subcutaneous fat thicknesses measured. The leg musculature was not influenced by increasing the thickness of subcutaneous fat. Based on this study and also with the requirements of the consumer market, it is recommended that the slaughter of animals occur with approximately $3.00 \mathrm{~mm}$ fat.
\end{abstract}

Keywords: females, muscularity, ultrasound, yield

Recebido em 5 de fevereiro de 2014

Aceito em 4 de setembro de 2014

E-mail: natalia-mora@hotmail.com 


\section{INTRODUÇÃO}

Os Estados do Mato Grosso e Mato Grosso do Sul possuem rebanho efetivo de ovinos de 972.162 mil cabeças, perfazendo $5,55 \%$ do rebanho nacional (Instituto..., 2011). Nesses, além de várias raças encontradas em outras regiões da federação, existe também o grupo genético de ovinos denominados "pantaneiros", oriundos de muitos cruzamentos ao longo de anos, que sofreram seleção natural às condições climáticas da região, tendo como principais ancestrais animais do Sul e Nordeste (Vargas Junior et al., 2011). Esses animais têm como características básicas a presença de lã, alta rusticidade, boa habilidade materna, cio ao longo do ano.

Adequações dos produtores desses ovinos ao sistema tecnológico de criação devem ser realizadas para obtenção de precocidade muscular e apropriada espessura de gordura das carcaças dos animais comercializados para tornálos mais atrativos. Nesse contexto, é de suma importância a realização de pesquisas acerca da ovinocultura no Brasil.

Como ferramenta de melhora de produtividade e demanda, a ultrassonografia pode ser utilizada em criações de ovinos, com intuito de garantir o momento ideal de abate de uma forma prática; pois, segundo Peres et al. (2010), melhora a predição da composição corporal ou de gordura na carcaça.

Embora a carcaça seja o principal produto da ovinocultura no Brasil, sua comercialização em cortes ainda não é amplamente definida devido à falta de padronização, por questões culturais e regionais, de regiões musculares, de acordo com a qualidade e valor.

Em relação às estimativas de predição da carcaça e dos cortes, a área de olho-de-lombo é considerada a melhor medida que representa a quantidade e distribuição das massas musculares, assim como da qualidade da carcaça. Os músculos de maturidade tardia são indicados para representar o índice mais confiável do desenvolvimento e tamanho do tecido muscular; assim, o Longissimus dorsi é o mais indicado, pois, além do amadurecimento tardio, é de fácil mensuração (Hashimoto et al., 2012) com o auxílio da ultrassonografia.
Outra ferramenta na predição da proporção muscular na carcaça de ovinos é o índice de musculosidade da perna, que é descrito como a profundidade média de um grupo de músculos que circundam o fêmur em relação ao comprimento desse osso (Purchas et al., 1991). Esse índice pode representar a relação músculo:osso, sendo maior quando houver grande quantidade de carne nas carcaças.

São escassas as informações sobre as características quantitativas de carcaça de cordeiras Pantaneiras em sistemas intensivos de produção, salientando o interesse de produtores na região Centro-Oeste pela criação, que vem ocorrendo de forma empírica.

O objetivo do estudo foi avaliar as características quantitativas da carcaça e o índice de musculosidade da perna de cordeiras Pantaneiras, abatidas com diferentes espessuras de gordura subcutânea.

\section{MATERIAL E MÉTODOS}

O experimento foi realizado na Fazenda Experimental de Iguatemi da Universidade Estadual de Maringá (UEM), Maringá, Paraná, de outubro de 2012 a janeiro de 2013.

Foram utilizadas 24 fêmeas ovinas, com aproximadamente 100 dias de idade e peso vivo médio de $16,24 \pm 1,78 \mathrm{~kg}$, todas do grupamento genético Pantaneiro, adquiridas em propriedades de sistema extensivo, na região Sudoeste do Estado do Mato Grosso, área de tensão biológica com transição do Pantanal, Cerrado e Floresta Amazônica.

Inicialmente os animais receberam antihelmíntico à base de Moxidectina como princípio ativo, e passaram por um período de 15 dias de adaptação à instalação e à dieta. Posteriormente foram pesados e as espessuras de gordura subcutânea, avaliadas por ultrassonografia, sendo distribuídos nos respectivos tratamentos, definidos como espessuras de gordura subcutânea no Longissimus dorsi, entre a $12^{\mathrm{a}}$ e a $13^{\mathrm{a}}$ costela, em 2,0;3,0 e 4,0 mm.

As cordeiras foram distribuídas em baias individuais cobertas, com área de $0,75 \mathrm{~m}^{2}$, com piso ripado suspenso. Durante todo o período experimental, receberam água à vontade e foram 
alimentadas com dieta completa (Tab. 1), misturada e peletizada, formulada para ganho de peso diário de $0,300 \mathrm{~kg}$ (National...,, 2007), fornecida uma vez ao dia no período da manhã, de maneira a proporcionar sobras de aproximadamente $10 \%$.

Tabela 1. Composição em $\mathrm{g} / \mathrm{kg}$ na matéria seca dos ingredientes e análise químicobromatológica da dieta experimental

\begin{tabular}{|c|c|}
\hline Item & $\begin{array}{c}\text { Composição } \\
(\mathrm{g} / \mathrm{kg})\end{array}$ \\
\hline Feno de aveia & 100,0 \\
\hline Grão de milho moído & 448,0 \\
\hline Farelo de soja & 150,0 \\
\hline Casca de soja & 150,0 \\
\hline Farelo de arroz & 100,0 \\
\hline Melaço em pó & 20,0 \\
\hline Cloreto de amônio & 20,0 \\
\hline Mistura mineral $^{1}$ & 10,0 \\
\hline Bacitracina de zinco & 02,0 \\
\hline Total & 1000,0 \\
\hline Matéria seca & 912,8 \\
\hline Proteína bruta & 162,4 \\
\hline Extrato etéreo & 42,1 \\
\hline Fibra em detergente neutro & 275,4 \\
\hline Fibra em detergente ácido & 138,6 \\
\hline Cinzas & 45,9 \\
\hline Cálcio & 02,8 \\
\hline Fósforo & 04,0 \\
\hline $\begin{array}{l}\text { Digestibilidade in vitro da } \\
\text { matéria seca }^{2}\end{array}$ & 782,5 \\
\hline Nutrientes digestíveis totais ${ }^{3}$ & 766,8 \\
\hline
\end{tabular}

Custo total da ração

${ }^{1}$ Níveis de garantia da mistura mineral por kg: Cálcio $220 \mathrm{~g}$, Fósforo 130g, Magnésio 25,5g, Enxofre 24g, Ferro $3.000 \mathrm{mg}$, Manganês $1.500 \mathrm{mg}$, Zinco $4.000 \mathrm{mg}$, Cobre $1.200 \mathrm{mg}$, Cobalto $280 \mathrm{mg}$, Iodo $260 \mathrm{mg}$, Selênio $30 \mathrm{mg}$ e Flúor $300 \mathrm{mg} .{ }^{2}$ Metodologia de Tilley e Terry (1963), adaptada para o uso do rúmen artificial, desenvolvido por Ankom ${ }^{\circledR}$, conforme descrito por Garman et al. (1997). ${ }^{3} \mathrm{NDT}$ estimado pela equação \% $\mathrm{NDT}=92,2-(1,12 \times \mathrm{FDA})$, descrita por Aldai et al. (2010).

As avaliações por ultrassonografia e pesagens foram realizadas a cada sete dias. Para obtenção da espessura de gordura subcutânea, foi utilizado um equipamento de ultrassom, marca "HONDA", modelo HS-1500 VET, com transdutor linear multifrequêncial de $50 \mathrm{~mm}$ de largura, utilizando frequência de 7,5 MHz. Para a realização das medidas, as cordeiras foram imobilizadas manualmente e, com auxílio de pente, foi feita a separação da lã nas áreas de medição, assim como aplicada a mucilagem para o melhor acoplamento do transdutor à pele.
A pressão da cabeça do transdutor foi mantida mínima para evitar a compressão da gordura, e todas as mensurações foram realizadas pelo mesmo técnico, do lado esquerdo, entre a $12^{\mathrm{a}}$ e a $13^{\mathrm{a}}$ costela, a quatro $\mathrm{cm}$ da linha mediana da coluna vertebral. Depois de capturada a imagem, a espessura da gordura subcutânea foi mensurada usando-se o cursor eletrônico do ultrassom.

Conforme as cordeiras atingiam a espessura de gordura pré-determinada de 2,0; 3,0 e 4,0 mm na avaliação quinzenal por ultrassonografia, as mesmas eram abatidas no dia seguinte às aferições, independentemente do peso.

Após $18 \mathrm{~h}$ em jejum de sólidos, os animais foram pesados para determinação do peso corporal ao abate, em seguida insensibilizados por meio de descarga de 220 Volts por 8 segundos, seguida pela sangria das veias jugulares e as artérias carótidas, esfola e retirada dos órgãos internos.

Durante a evisceração, o aparelho gastrintestinal foi esvaziado para obtenção do peso do corpo vazio (PCV), determinado pelo peso corporal ao abate (PCA) subtraído do conteúdo gastrintestinal. As carcaças foram pesadas para obtenção do peso da carcaça quente (PCQ) e, após $24 \mathrm{~h}$ a $4^{\circ} \mathrm{C}$ em câmara frigorífica, foi obtido o peso da carcaça fria (PCF). Com os pesos, obtiveram-se o rendimento comercial da carcaça $\left(\mathrm{RCC}=\mathrm{PCF} / \mathrm{PCA}^{*} 100\right)$ e o rendimento verdadeiro da carcaça $\left(\mathrm{RVC}=\mathrm{PCQ} / \mathrm{PCV}^{*} 100\right)$.

Foi medido o comprimento interno da carcaça (CIC), sendo a distância máxima entre o bordo interior da sínfise isquiopubiana e o bordo anterior da primeira costela em seu ponto médio; a largura da garupa (LG) pela medida máxima entre os trocânteres de ambos os fêmures, tomada com compasso; e comprimento da perna (CP) pela distância entre o períneo e o bordo anterior da superfície articular tarsometatarsiana, sendo utilizados para calcular o índice de compacidade da carcaça (ICC), ICC $=\mathrm{PCF} / \mathrm{CIC}$, e o índice de compacidade da perna (ICP), $\mathrm{ICP}=\mathrm{LG} / \mathrm{CP}$.

As meias carcaças da direita foram seccionadas em cinco cortes para cálculo do rendimento de cada corte em relação à meia carcaça, sendo: pescoço, entre a $5^{\mathrm{a}}$ e a $6^{\mathrm{a}}$ vértebra cervical; paleta, em que foi desarticulada a escápula, liberando a peça da carcaça; costilhar, entre a $1^{\mathrm{a}}$ 
e a $13^{\mathrm{a}}$ vértebra torácica; lombo, entre a $1^{\mathrm{a}}$ e a $6^{\mathrm{a}}$ vértebra lombar; e perna, entre a última vértebra lombar e a primeira vértebra sacra.

A partir do corte denominado lombo (no músculo $L$. dorsi entre a última vértebra torácica e a primeira lombar), tomou-se a área transversal em transparência para posterior determinação da área de olho de lombo, através do programa ImageJ ${ }^{\circledR} 1.46$ r. Na mesma secção, sob o corte lombo, utilizando-se paquímetro, foram feitas quatro medidas: A - largura máxima do músculo; B - profundidade do músculo; C - espessura de gordura subcutânea sobre o músculo (continuação de B); J - espessura de gordura subcutânea máxima no perfil do lombo (continuação de A). Os lombos foram então identificados, embalados individualmente em saco plástico, armazenados em freezer a $-18^{\circ} \mathrm{C}$, sendo posteriormente dissecados para determinação da proporção de músculo:gordura:osso.

A perna foi dissecada e foram separados os seguintes grupos de tecidos: gordura total, músculos e ossos. O índice de musculosidade da perna foi calculado pela fórmula descrita por Purchas et al. (1991): índice de musculosidade = $[(\mathrm{PM} 5 / \mathrm{CF}) / \mathrm{CF}]^{0.5}$, em que PM5 é o peso (g) dos cinco músculos que recobrem o fêmur (bíceps femoral, semitendinoso, adutor, semimembranoso e quadríceps femoral); e o CF é o comprimento $(\mathrm{cm})$ do fêmur.

Para realização das análises estatísticas, o delineamento experimental utilizado foi inteiramente ao acaso com três tratamentos 2,0; 3,0 e $4,0 \mathrm{~mm}$ de espessura de gordura subcutânea e oito repetições, submetidos à análise de variância, e as médias dos resultados foram comparadas pelo teste Tukey a 5\% de probabilidade com auxílio do software Statistical Analysis System (Statistical..., 2001).

\section{RESULTADOS E DISCUSSÃO}

$\mathrm{Na}$ Tabela 2 são ilustradas as variáveis quantitativas das carcaças de cordeiras abatidas com diferentes espessuras de gordura subcutânea. $\mathrm{O}$ peso corporal ao abate (PCA) e o vazio (PCV) aumentaram conforme acréscimos na espessura de gordura, diferindo em mais de $6 \mathrm{~kg}$ entre os tratamentos, sendo justificado pela necessidade de maiores pesos corporais para alcançar valores superiores de deposição de gordura, pois esse tecido desenvolve-se mais na fase de terminação (Stydom et al., 2009).

Tabela 2. Médias e desvios padrão para características quantitativas de carcaça de cordeiras Pantaneiras abatidas com diferentes espessuras de gordura subcutânea

\begin{tabular}{lcccc} 
& \multicolumn{3}{c}{ Espessura de gordura } & \multirow{2}{*}{ Item } \\
\cline { 2 - 4 } & $2,0 \mathrm{~mm}$ & $3,0 \mathrm{~mm}$ & $4,0 \mathrm{~mm}$ & 9,16 \\
\hline PCA & $20,64 \pm 0,85 \mathrm{c}$ & $26,77 \pm 0,80 \mathrm{~b}$ & $32,12 \pm 0,91 \mathrm{a}$ & 11,49 \\
PCV & $17,63 \pm 0,96 \mathrm{c}$ & $23,75 \pm 0,91 \mathrm{~b}$ & $30,93 \pm 1,03 \mathrm{a}$ & 10,72 \\
PCQ & $9,47 \pm 0,48 \mathrm{c}$ & $13,12 \pm 0,45 \mathrm{~b}$ & $15,63 \pm 0,51 \mathrm{a}$ & 11,32 \\
PCF & $9,33 \pm 0,50 \mathrm{c}$ & $12,82 \pm 0,47 \mathrm{~b}$ & $15,32 \pm 0,53 \mathrm{a}$ & 7,82 \\
RVC & $53,96 \pm 1,48 \mathrm{a}$ & $55,48 \pm 1,40 \mathrm{a}$ & $53,31 \pm 1,68 \mathrm{a}$ & 4,65 \\
RCC & $45,15 \pm 0,76 \mathrm{a}$ & $46,60 \pm 0,72 \mathrm{a}$ & $47,69 \pm 0,82 \mathrm{a}$ & 10,15 \\
ICC & $0,17 \pm 0,008 \mathrm{~b}$ & $0,23 \pm 0,007 \mathrm{a}$ & $0,25 \pm 0,008 \mathrm{a}$ & 8,52 \\
ICP & $0,47 \pm 0,01 \mathrm{a}$ & $0,44 \pm 0,01 \mathrm{a}$ & $0,44 \pm 0,01 \mathrm{a}$ & \\
\hline
\end{tabular}

PCA: Peso corporal ao abate (kg); PCV: peso corporal vazio (kg); PCQ: Peso carcaça quente (kg); PCF: Peso carcaça fria (kg); PCV: Peso corporal vazio (kg); RCC: Rendimento comercial de carcaça (\%); RVC: Rendimento verdadeiro de carcaça (\%); ICC: Índice de compacidade da carcaça $(\mathrm{kg} / \mathrm{cm})$; ICP: Índice de compacidade da perna; CV: coeficientes de variação. Médias seguidas de letras iguais não diferem no teste Tukey $5 \%$.

Para peso de carcaça quente (PCQ) e fria (PCF), houve diferença entre os tratamentos, com maiores valores constatados para os animais abatidos com 4,0mm de espessura de gordura, seguidos dos de 3,00 e posteriormente $2,00 \mathrm{~mm}$, semelhante ao ocorrido com o PCA e PCV. Sabe-se que normalmente o peso de carcaça possui correlação positiva com o peso corporal. Esse comportamento já foi constatado outrora por Nour et al. (1983), ao concluírem que, para cada quilograma a mais no peso corporal, tem-se aumento de $0,74 \mathrm{~kg}$ no peso de carcaça. 
As espessuras de gordura não influenciaram os rendimentos de carcaça, verdadeiro e comercial, provavelmente porque os tecidos corporais e os órgãos desenvolvem-se igualmente dentro de uma amplitude de maturidade corporal. Resultados de semelhança também foram observados por Amaral et al. (2011) ao trabalharem com cordeiros Santa Inês abatidos com diferentes espessuras de gordura $(2,0 ; 2,5$; $3,0 \mathrm{~mm})$.

O índice de compacidade da carcaça referência ao desenvolvimento dos tecidos corporais em relação ao seu comprimento: Para esse índice, as carcaças oriundas dos tratamentos 4,00 e $3,00 \mathrm{~mm}$ de espessura de gordura foram superiores aos valores das cordeiras abatidas com 2,00mm. Isso se justifica pela curva de crescimento corporal baseada nos desenvolvimentos diferentes entre os tecidos corporais, e sabendo que o músculo desenvolvese de forma intermediária, e a gordura, mais tardiamente; os animais do tratamento $2,00 \mathrm{~mm}$ tinham menor quantidade desses tecidos em relação a seu comprimento. Pinto (2009) obteve média de $0,25 \mathrm{~kg} / \mathrm{cm}$ trabalhando com o mesmo grupo genético.
O índice de compacidade de perna não apresentou diferença para os animais abatidos com 2,0;3,0 e 4,0mm de gordura subcutânea. Gualda (2011) não encontrou efeito para essa variável com cordeiros tanto para grupo racial (Santa Inês e 1/2 Dorper-Santa Inês) como para espessura de gordura $(2,0 ; 3,0$ e 4,0mm) com média de 0,53 .

$\mathrm{Na}$ Tabela 3, encontram-se os rendimentos dos cortes comerciais em relação à meia carcaça. As porcentagens dos cortes pescoço, paleta e costilhar não diferiram entre os tratamentos. Em relação ao lombo, os animais abatidos com 4,00 $\mathrm{mm}$ de espessura de gordura apresentaram maior porcentagem desse corte em relação aos animais abatidos com 2,00 e $3,00 \mathrm{~mm}$. A porcentagem de perna foi maior para os animais abatidos com menores espessuras de gordura $(2,00$ e $3,00 \mathrm{~mm})$. Pinheiro et al. (2007) justificam esse comportamento de desenvolvimento corporal ao descreverem que os aspectos de desenvolvimento tecidual de cada região anatômica devem ser analisados isoladamente, pois o crescimento é precoce na paleta, intermediário, na perna, e tardio, no lombo.

Tabela 3. Rendimento $(\%)$ de cortes comerciais da meia carcaça direita de cordeiras Pantaneiras abatidas com diferentes espessuras de gordura subcutânea

\begin{tabular}{llllc}
\hline & \multicolumn{3}{c}{ Espessura de gordura } & \multirow{2}{*}{ CV $(\%)$} \\
\cline { 2 - 4 } Item $(\%)$ & \multicolumn{1}{c}{$2,0 \mathrm{~mm}$} & $3,0 \mathrm{~mm}$ & $4,0 \mathrm{~mm}$ & 16,54 \\
Pescoço & $5,20 \pm 0,30 \mathrm{a}$ & $5,23 \pm 0,29 \mathrm{a}$ & $5,22 \pm 0,32 \mathrm{a}$ & 20,87 \\
Paleta & $18,75 \pm 1,26 \mathrm{a}$ & $17,72 \pm 1,19 \mathrm{a}$ & $17,12 \pm 1,35 \mathrm{a}$ & 13,50 \\
Lombo & $11,76 \pm 0,63 \mathrm{~b}$ & $12,71 \pm 0,59 \mathrm{~b}$ & $15,32 \pm 0,62 \mathrm{a}$ & 7,33 \\
Costilhar & $27,95 \pm 0,75 \mathrm{a}$ & $28,83 \pm 0,71 \mathrm{a}$ & $29,89 \pm 0,80 \mathrm{a}$ & 6,49 \\
Perna & $36,34 \pm 0,8 \mathrm{a}$ & $36,11 \pm 0,75 \mathrm{a}$ & $31,92 \pm 0,85 \mathrm{~b}$ & \\
\hline
\end{tabular}

$\mathrm{CV}$ : coeficientes de variação. Médias seguidas de letras iguais não diferem no teste Tukey $5 \%$.

Houve diferença para condição corporal em relação às medidas de gordura subcutânea (Tab. 4). Animais com 2,0mm tiveram uma menor condição corporal, pelo fato de ficarem menos tempo confinados, comparados aos outros tratamentos, pois essa condição é mensurada principalmente com base no desenvolvimento muscular e adiposo do animal.

As espessuras de gordura subcutânea influenciaram a área de olho de lombo (AOL). Como evidenciado, o músculo Longissimus dorsi é de maturidade tardia, sendo indicado para representar o desenvolvimento e o tamanho do tecido muscular, confirmando o resultado encontrado neste experimento, em que a AOL foi maior em animais com maior tempo de confinamento $(3,0$ e $4,0 \mathrm{~mm}$ de espessura de gordura subcutânea). Esses valores corroboram Gualda (2011), que, ao trabalhar com cordeiros da raça Santa Inês e 1/2 Dorper-Santa Inês, abatidos com diferentes espessuras de gordura subcutânea $(2,0 ; 3,0$ e 4,0mm), verificou maior AOL para cordeiros abatidos com $4,0 \mathrm{~mm}$ de espessura de gordura, quando comparados com animais abatidos com 2,0 e $3,0 \mathrm{~mm}$. 
Características de carcaça...

Tabela 4. Médias e desvio padrão das medidas do lombo de cordeiras abatidas com diferentes espessuras de gordura

\begin{tabular}{|c|c|c|c|c|}
\hline \multirow[b]{2}{*}{ Item } & \multicolumn{3}{|c|}{ Espessura de gordura } & \multirow[b]{2}{*}{$\mathrm{CV}(\%)$} \\
\hline & $2,0 \mathrm{~mm}$ & $3,0 \mathrm{~mm}$ & $4,0 \mathrm{~mm}$ & \\
\hline $\mathrm{CC}$ & $2,15 \pm 0,13 b$ & $3,06 \pm 0,12 \mathrm{a}$ & $3,14 \pm 0,14 \mathrm{a}$ & 13,47 \\
\hline $\mathrm{AOL}$ & $8,92 \pm 0,75 b$ & $10,36 \pm 0,71 \mathrm{ab}$ & $11,78 \pm 0,81 \mathrm{a}$ & 20,69 \\
\hline Medida $\mathrm{C}_{\text {(us) }}$ & $2,03 \pm 0,06 \mathrm{c}$ & $3,04 \pm 0,06 b$ & $3,89 \pm 0,05 \mathrm{a}$ & 6,12 \\
\hline Medida $\mathrm{C}_{(\mathrm{p})}$ & $1,91 \pm 0,16 \mathrm{c}$ & $3,05 \pm 0,15 b$ & $4,10 \pm 0,17 \mathrm{a}$ & 14,25 \\
\hline Medida J & $4,25 \pm 0,56 b$ & $5,63 \pm 0,53 b$ & $8,10 \pm 0,06 \mathrm{a}$ & 26,95 \\
\hline Medida A & $23,27 \pm 1,34 b$ & $27,23 \pm 1,26 \mathrm{ab}$ & $30,23 \pm 1,43 a$ & 14,16 \\
\hline Medida B & $44,99 \pm 1,37 b$ & $48,36 \pm 1,29 \mathrm{ab}$ & $50,56 \pm 1,46 a$ & 8,105 \\
\hline Músculo (\%) & $58,91 \pm 2,3^{\mathrm{a}}$ & $53,9 \pm 2,17 \mathrm{a}$ & $49,34 \pm 2,46 b$ & 12,35 \\
\hline Gordura (\%) & $24,87 \pm 1,96 b$ & $34,60 \pm 1,85 \mathrm{a}$ & $39,33 \pm 2,04 a$ & 16,93 \\
\hline Osso (\%) & $16,21 \pm 1,04^{\mathrm{a}}$ & $11,51 \pm 0,98 b$ & $11,33 \pm 1,11 \mathrm{~b}$ & 20,23 \\
\hline
\end{tabular}

CC: Condição Corporal; AOL: Área de olho de lombo; C(p): medida feita com paquímetro; C(us): medida feita com ultrassonografia. CV: coeficientes de variação. Médias seguidas de letras iguais não diferem no teste Tukey $5 \%$.

As medidas de espessura de gordura subcutânea obtidas por paquímetro (Medida $\mathrm{C}_{(\mathrm{p})}$ ) e por ultrassonografia (Medida $\mathrm{C}_{(\mathrm{us})}$ ) diferiram entre os tratamentos, obtendo-se maiores valores de espessura de gordura subcutânea para cordeiras abatidas com 4,0mm. As diferenças nas mensurações das Medidas $\mathrm{C}$ se justificam pela base dos princípios de crescimento relativo que, para ovinos, a partir de 3-4 meses de idade, temse o aumento da deposição de gordura corporal (Hammond, 1932). Gualda (2011) e Amaral et al. (2011), ao trabalharem com cordeiros Santa Inês, $1 / 2$ Dorper-Santa Inês e $1 / 2$ White DorperSanta Inês abatidos com diferentes espessuras de gordura subcutânea, não encontraram diferença entre a espessura de gordura subcutânea obtida por paquímetro e por ultrassonografia, confirmando a confiabilidade das medidas obtidas pela ultrassonografia.

Para aplicabilidade comercial, o aumento da espessura de gordura subcutânea é importante para obtenção de melhor acabamento da carcaça, além de protegê-la contra a queima por resfriamento e perdas de água.

As médias para a Medida J, Medida A e Medida B diferiram entre os tratamentos, sendo cordeiras abatidas com 4,0mm de espessura de gordura subcutânea com maiores valores, que demonstra à progressiva e simultânea deposição dos tecidos musculares e adiposos ao longo tempo de confinamento.

Foram observadas diferenças nas proporções de músculo, osso e gordura entre as espessuras de gordura subcutânea avaliadas. Cordeiras abatidas com 4,0mm de espessura de gordura apresentaram menor proporção de músculo comparação com os demais tratamentos, corroborando os dados encontrados por Gualda (2011). Isso é explicado pelo fato de, ao atingirem o ponto médio da curva de crescimento, o desenvolvimento do músculo diminui e a deposição de gordura aumenta, tendo assim uma inversão das proporções de músculo:gordura (Purchas et al., 1991).

Os animais abatidos com 4,0mm de espessura de gordura apresentaram menor proporção de ossos, o tecido mais precoce do que o muscular no início da vida dos cordeiros, sofrendo desaceleração à medida que o animal envelhece. Stydom et al. (2009), ao analisarem a composição dos principais cortes de carcaças de cordeiros, com cinco diferentes escores de gordura, observaram que as proporções de osso diminuíram significativamente com o aumento da gordura.

Encontram-se na Tabela 5 a composição e o índice de musculosidade da perna de cordeiras abatidas 
com diferentes espessuras de gordura subcutânea. Houve um aumento de peso das pernas $(\mathrm{P} \leq 0,05)$ quando se aumentou a espessura de gordura, evidenciando cordeiras do tratamento com 4,0mm, que ficaram mais tempo confinadas. Na dissecação, tanto os cinco músculos envolvidos no fêmur como a quantidade de músculo total na perna tiveram influência significativa, sendo menores as médias de peso para os animais abatidos com 2,0mm de espessura de gordura.

Tabela 5. Composição e índice de musculosidade da perna de cordeiras Pantaneiras abatidas com diferentes espessuras de gordura subcutânea

\begin{tabular}{|c|c|c|c|c|}
\hline \multirow[b]{2}{*}{ Característica } & \multicolumn{3}{|c|}{ Espessura de gordura subcutânea } & \multirow[b]{2}{*}{$\mathrm{CV} \%$} \\
\hline & $2,0 \mathrm{~mm}$ & $3,0 \mathrm{~mm}$ & $4,0 \mathrm{~mm}$ & \\
\hline Peso da perna $(\mathrm{kg})$ & $1,66 \pm 0,18 b$ & $1,92 \pm 0,18 \mathrm{ab}$ & $2,51 \pm 0,18 \mathrm{a}$ & 23,015 \\
\hline \multicolumn{5}{|l|}{ Músculo (g) } \\
\hline Músculo total & $1,04 \pm 0,04 \mathrm{c}$ & $1,33 \pm 0,04 b$ & $1,49 \pm 0,04 \mathrm{a}$ & 8,58 \\
\hline Semimembranoso & $0,14 \pm 0,001 b$ & $0,18 \pm 0,001 \mathrm{ab}$ & $0,18 \pm 0,001 \mathrm{a}$ & 18,56 \\
\hline Semitendinoso & $0,06 \pm 0,006 b$ & $0,07 \pm 0,007 b$ & $0,12 \pm 0,007 \mathrm{a}$ & 22,72 \\
\hline Bíceps femoral & $0,14 \pm 0,008 b$ & $0,19 \pm 0,005 a$ & $0,19 \pm 0,005 a$ & 11,92 \\
\hline Quadríceps femoral & $0,21 \pm 0,010 b$ & $0,26 \pm 0,009 a$ & $0,29 \pm 0,009 a$ & 10,34 \\
\hline Adutor & $0,06 \pm 0,005 b$ & $0,07 \pm 0,005 \mathrm{ab}$ & $0,08 \pm 0,005 \mathrm{a}$ & 18,67 \\
\hline Gordura total $(\mathrm{g})$ & $0,32 \pm 0,11 b$ & $0,45 \pm 0,11 \mathrm{a}$ & $0,46 \pm 0,11 \mathrm{a}$ & 73,58 \\
\hline \multicolumn{5}{|l|}{ Osso da perna (g) } \\
\hline Osso total & $0,30 \pm 0,29 a$ & $0,35 \pm 0,27 a$ & $0,33 \pm 0,29 a$ & 23,51 \\
\hline Fêmur & $0,145 \pm 0,06 a$ & $0,220 \pm 0,06 \mathrm{a}$ & $0,191 \pm 0,06 a$ & 45,47 \\
\hline Comprimento $(\mathrm{cm})$ & $17,42 \pm 0,73 \mathrm{a}$ & $17,75 \pm 0,68 \mathrm{a}$ & $18,6 \pm 0,73 a$ & 10,75 \\
\hline Relação músculo:osso & $4,38 \pm 0,75 \mathrm{a}$ & $5,11 \pm 0,71 \mathrm{a}$ & $6,12 \pm 0,75 a$ & 38,32 \\
\hline Musculosidade & $0,35 \pm 0,02 \mathrm{a}$ & $0,37 \pm 0,19 \mathrm{a}$ & $0,37 \pm 0,02 \mathrm{a}$ & 14,9 \\
\hline
\end{tabular}

$\mathrm{CV}$ : coeficientes de variação. Médias seguidas de letras iguais não diferem no teste Tukey $5 \%$.

A composição tecidual da perna é de grande importância na avaliação da qualidade da carcaça, pois apresenta altos índices de correlação, quanto às suas proporções de músculo, osso e gordura, com a carcaça inteira de ovinos (Cézar e Souza, 2010). Cordeiras abatidas com 2,0mm de espessura de gordura subcutânea obtiveram menos quantidade de gordura total, porém as médias não diferiram entre os tratamentos. O tecido adiposo é o que possui maior variabilidade no animal, tanto quantitativamente como em função da sua distribuição (Rosa et al., 2005), e esses depósitos de gordura podem ser influenciados principalmente pelo nível de energia contido na dieta, tempo de ingestão dessa dieta e o sexo, sendo que fêmeas possuem maior depósito comparando-se aos machos.

O peso do osso total, peso do fêmur e seu comprimento não diferiram entre os tratamentos.
Não foi observada diferença significativa na relação músculo:osso com um valor médio de 5,20. Esses resultados podem ter sido influenciados com comprimento e peso do osso que não foram influenciados pelos tratamentos. Segundo Moreno et al. (2010), o índice de musculosidade da perna representa bem a relação músculo:osso, sendo maior quando maior for a quantidade de carne nas carcaças, em função da dimensão do esqueleto, podendo variar em virtude de ossos mais leves, em vez de músculos mais pesados.

Medidas de musculosidade podem, também, não diferir, mesmo quando há diferenças na quantidade de músculo, decorrentes das variações no comprimento de osso, por causa da idade, considerando-se uma mesma raça (Silva Sobrinho et al., 2005). 
A musculosidade da perna não foi influenciada com o aumento da espessura da gordura subcutânea; isso se deve à menor aptidão do agrupamento Pantaneiro para a produção de carne. Entretanto as médias encontradas foram aproximadas ao encontrado por Andrade (2013), Costa et al. (2010) e Cunha et al. (2008) utilizando raças nativas brasileiras.

\section{CONCLUSÃO}

Com base neste estudo e também pelas exigências do mercado consumidor, recomendase $\mathrm{o}$ abate de animais com aproximadamente $3,00 \mathrm{~mm}$ de gordura, pois, mesmo não havendo diferenças entre os tratamentos para os rendimentos de carcaça, para o ICC e \% de gordura os maiores valores foram observados nos ovinos abatidos com 3,00 e 4,00mm; contudo, neste último tratamento houve maior redução na porcentagem de musculatura total da carcaça.

\section{REFERÊNCIAS}

ALDAI, N.; DUGAN, M.E.R.; KRAMER, J.K.G. et al. Trans-18:1 and conjugated linoleic acid profiles after the inclusion of buffer, sodium sesquicarbonate, in the concentrate of finishing steers. Meat Sci., v.84, p.735-741, 2010.

AMARAL, R.M.; MACEDO, F.A.F.; ALCALDE, C.R. et al. Desempenho produtivo e econômico de cordeiros confinados abatidos com três espessuras de gordura. Rev. Bras. Saúde Prod. Anim., v.12, p.155-165, 2011.

ANDRADE, M.G.L.P. Características $d a$ carcaça e qualidade da carne de cordeiros Santa Inês e Morada Nova em diferentes pesos ao abate. 2013. 100f. Tese (Doutorado em Zootecnia) - Universidade Federal da Paraíba, Areias.

CÉZAR, M.F.; SOUZA, W.H. Carcaças ovinas e caprinas: obtenção, avaliação e classificação. Uberaba: Editora Agropecuária Tropical, 2007. $147 \mathrm{p}$.

COSTA, R.G.; ARAUJO FILHO, J.T.; SOUZA, W.H. et al. Effect of diet and genotype on carcass characteristics of feedlot hair sheep. Rev. Bras. Zootec. [online]. v.39, p.2763-2768, 2010.
CUNHA, M.G.G.; CARVALHO, F.F.R.; GONZAGA NETO, S. et al. Características quantitativas de carcaça de ovinos Santa Inês confinados alimentados com rações contendo diferentes níveis de caroço de algodão integral. Rev. Bras. Zootec., v.37, p.1112-1120, 2008.

GARMAN, C.L.; HOLDEN, L.A.; KANE, H.A. Comparison of in vitro dry matter digestibility of nine feedstuffs using three methods of analysis. J. Dairy Sci., v.80, p.260, 1997.

GUALDA, T.P. Desempenho e características de carcaça de cordeiros com três espessuras de gordura subcutânea no lombo. 2011. 46f. Dissertação (Mestrado em Zootecnia) Universidade Estadual de Maringá, Maringá.

HAMMOND, J. Growth and development of mutton qualities in the sheep. Edinburgh, UK: Oliver and Boyd, 1932. 595p.

HASHIMOTO, J.H.; OSÓRIO, J.C.S.; OSÓRIO, M.T.M. et al. Qualidade de carcaça, desenvolvimento regional e tecidual de cordeiros terminados em três sistemas. Rev. Bras. Zootec., v.41, p.438-448, 2012.

INSTITUTO Brasileiro De Geografia E Estatítica - IBGE. 2011. Rio de Janeiro, 2011. Disponível em: <http://www.ibge.gov.br/ estadosat/index.php>. Acessado em: 7 mar. 2013.

MORENO, G.M.B.; SOBRINHO, A.G.S.; LEÃO, A.G. et al. Rendimentos de carcaça, composição tecidual e musculosidade da perna de cordeiros alimentados com silagem de milho ou cana-de-açúcar em dois níveis de concentrado. Arq. Bras. Med. Vet. Zootec., v.62, p.686-695, 2010.

NATIONAL Research Council - NRC. Nutrient Requirements of Small Ruminants: Sheep, Goats, Cervids, and New World Camelids. Washington, DC.: National Academy Press., 2007. 384 p.

NOUR, A.Y.M.; THONNEY, M.L.; STOUFFERR WHITE, W.R.C. et al. Changes in carcass weight and characteristics with increasing weight of large and small cattle. $J$. Animal Sci., v.57, p.1154-1165, 1983.

PERES, A.M.; DIAS, L.G.; JOY, M.; TEIXEIRA, A. Assessment of goat carcass fat composition using ultrasound technology and multiple multivariate prediction models. $J$. Animal Sci., v.88, p.572-580, 2010. 
PINHEIRO, R.S.B.; SILVA SOBRINHO, A.G.; MARQUES, C.A.T.; YAMAMOTO, S.M. Biometria in vivo e da carcaça de cordeiros confinados. Pesq. Agrop. Bras., v.56, p.955-958, 2007.

PINTO, G.S. Avaliação quantitativa da carcaça de cordeiros filhos de ovelhas pantaneiras acasaladas com carneiros pantaneiros, Santa Inês e Texel. 2009. 61f. Dissertação (Mestrado em Produção e Gestão Agroindustrial) Universidade Anhanguera, Campo Grande.

PURCHAS, R.W.; DAVIES, A.S.; ABDUKKAH, A.Y. An objective measure of muscularity: changes with animal growth and differences between genetic lives of southdown sheep. Meat Sci., v.30, p.81-94, 1991.

ROSA, G.T.; PIRES, C.C.; SILVA, J.H.S. Crescimento alométrico de osso, músculo e gordura em cortes da carcaça de cordeiros Texel segundo os métodos de alimentação e peso de abate. Cienc. Rural, v.35, p.870-876, 2005.
SAS - STATISTICAL ANALYSIS SYSTEM.

User's guide: software version 8.1. 4th ed. Cary: SAS Institute, 2001.

SILVA SOBRINHO, A.G.; PURCHAS, R.N.; KADIM, I.T. Musculosidade e composição da perna de ovinos de diferentes genótipos e idades de abate. Pesq. Agrop. Bras., v.40, p.1129-1134, 2005.

STYDOM, P.E.; VAN HEERDEN, S.M.; SCHONFELDT, H.C. et al. The influence of fat score and fat trimming on primal cut composition of South African Lamb. South Afri. J. Anim. Sci., v.3, p.233-242, 2009.

TILLEY, J.M.A.; TERRY, R.A. A two stage technique for the "in vitro" digestion of forage crop. J. British Grass. Soc., v.18, p.104-111, 1963.

VARGAS JUNIOR, F.M.; MARTINS, C.F.; SOUZA, C.C. et al. Avaliação Biométrica de Cordeiros Pantaneiros. Rev. Agrar. v.4, p.60-65, 2011. 